\title{
Supernatants from lymphocytes stimulated with Bacillus Calmette-Guerin can modify the antigenicity of tumours and stimulate allogeneic T-cell responses
}

\author{
WM Liu",', DW Fowler', AM Gravett', P Smith' and AG Dalgleish' \\ 'Department of Oncology, Division of Clinical Sciences, St George's, University of London, 2nd floor, Jenner Wing, London SWI 7 ORE, UK
}

\begin{abstract}
BACKGROUND: Reduced expression of class I human leucocyte antigens (HLAI) is often a mechanism by which tumours evade surveillance by the host immune system. This is often associated with an immune function that is unable to mount appropriate responses against disease, which can result in a state that favours carcinogenesis.

METHODS: In the current study, we have explored the effects of Bacillus Calmette-Guerin (BCG) on the cytokine output of leucocytes, which is a key determinant in generating antitumour action, and have also assessed the effect of these cytokine cocktails on HLAI expression in solid tumour cell lines.

RESULTS: BCG potently activated a broad range of leucocytes, and also enhanced the production of cytokines that were Th,-predominant. Supernatants from BCG-treated leucocytes significantly increased the expression of HLAI on the surface of cancer cell lines, which correlated with increased cytolytic T-cell activity. We also showed that the increased HLAI expression was associated with activation of intracellular signalling pathways, which was triggered by the increases in the Th,-cytokines interferon- $\gamma$ and tumour necrosis factor- $\alpha$, as counteracting their effects negated the enhancement.

CONCLUSION: These studies reaffirm the role of BCG as a putative immunotherapy through their cytokine-modifying effects on leucocytes and their capacity to enhance tumour visibility.

British Journal of Cancer (201I) 105, 687-693. doi:I0.1038/bjc.2011.306 www.bjcancer.com

Published online 9 August 2011

(c) 201 I Cancer Research UK
\end{abstract}

Keywords: HLAI; BCG; immunovisibility; immunotherapy; cytokines

It has been known for sometime that tumours possess antigens, which can be targeted by intrinsic immune processes as part of disease clearance. These tumour-associated antigens can be presented in complex with the human leucocyte antigen (HLA)-1 on their surface, which can evoke cytolytic immune responses (Yang and Yi, 2010). The principle of enhancing cell killing underscores immunotherapy for a variety of neoplasia. However, successful incorporations into clinical treatment strategies of approaches that exploit this effect have been limited (Copier et al, 2011). These have been due, in part, to deficiencies in the response of the immune system to tumour that have many causes; reasons have included (i) reduced immunovisibility of tumours, (ii) desensitised/anergic immune system and (iii) inappropriate immune reaction to tumours.

Specific immunity is broadly categorised as being cell-mediated or humoral, the initiation of which determines the suitability of the immune response. They can be distinguished by the types of cytokines produced by CD $4+\mathrm{T}$-cells, as cell-mediated immunity is often defined by the presence of $\mathrm{T}$-cells producing $\mathrm{Th}_{1}$ cytokines such as interleukin (IL) 2 , interferon (IFN)- $\gamma$ and tumour necrosis factor (TNF)- $\alpha$; while humoral immunity has a profile rich in $\mathrm{Th}_{2}$ cytokines such as IL4, IL6 and IL10 (Abbas et al, 1996). These

*Correspondence: DrWM Liu; E-mail: w.liu@sgul.ac.uk Received 6 June 2011; revised 12 July 2011; accepted I5 July 201।; published online 9 August 2011 cytokines have divergent functions, and determine the nature of the response. Generally, a $\mathrm{Th}_{1}$-dominant response is more effective against intracellular pathogens, while a $\mathrm{Th}_{2}$-response is directed more towards external stimuli. It is important that the proper type of immunity is triggered to ensure an effective response. Indeed, a $\mathrm{Th}_{1}$-response would favour the potentiation of a CD8 $+\mathrm{T}$-cell cytotoxic response against tumour (Dunn et al, 2002), whereas a $\mathrm{Th}_{2}$-response would antagonise this effect. Furthermore, a $\mathrm{Th}_{2}$ dominant profile is often seen in cancer patients that may favour tumour growth (Ellyard et al, 2007).

Treatment strategies that include systemic immune stimulation in its armoury would be powerful tools against cancer; and indeed, our group has focused on developing immunotherapies that follow such a course. Of particular interest has been our work with mycobacteria (Hrouda et al, 1998; Maraveyas et al, 1999; Nicholson et al, 2003). Stimulating an immune response using mycobacterium preparations such as Bacillus Calmette-Guerin (BCG) and Mycobacterium vaccae can enhance the cytotoxic response directed against some tumours. Furthermore, the mechanism of BCG may be mediated by $\mathrm{Th}_{1}$ cytokines (Orme et al, 1993), which evoke potent cell-mediated immune reactions against diseases. Apart from this effect, modifications to cytokine production by T-cells and other peripheral-blood mononuclear cells (PBMCs) may have other effects. For example, we have previously shown that some chemotherapies possess immunemodulatory character (Liu et al, 2010, 2011). This is manifest as an ability to upregulate HLA1 expression on tumours directly. As 
HLA1 expression on tumour cells can be induced by stimulation with type I interferons (Seliger et al, 2008), the effects that BCG had on cytokine production could provide a way that it could modify tumours to make them more antigenic. As cytotoxic T-cells are restricted by HLA1 and kill tumour cells expressive of them, the restoration of HLA1 expression could reinitialise immune visibility to these tumour cells and lead to an enhanced immunocytolytic response.

Consequently, as part of our ongoing research activities, we have worked on the hypothesis that BCG alters the cytokines produced by PBMCs that leads to a greater antigenicity of tumour cells and thus an enhanced cytotoxic immune response. Supplementary to this, we have also explored the role that signalling pathways have on this process.

\section{MATERIALS AND METHODS}

\section{Cell culture}

The human cancer cell lines A549 (lung), HCT116 (colon), MCF7 (breast) and MIAPaCa2 (pancreas) were obtained from the Cancer Research UK Cell Production Laboratories. The KM cell line was developed in house from a patient with melanoma. All cells were maintained in culture medium supplemented with $10 \%(\mathrm{v} / \mathrm{v})$ foetal bovine serum (FBS), $2 \mathrm{~mm}$ L-glutamine and $1 \%$ penicillin/ streptomycin (basal culture medium). All cell lines were incubated in a humidified atmosphere with $5 \% \mathrm{CO}_{2}$ in air at $37^{\circ} \mathrm{C}$, and discarded when the passage number exceeded 15.

Peripheral-blood mononuclear cells were isolated from whole blood or from the residue product of leucoreduction of whole blood from pathologically healthy donors (National Blood Service, London, UK) using Histopaque-1077 (Sigma Ltd, Dorset, UK). The mononuclear fraction was harvested and red blood cell contamination removed by incubation in hypotonic ammonium chloride. Cells were washed in phosphate buffered saline (PBS) and platelet contamination removed by centrifugation at $200 \mathrm{~g}$ for $10 \mathrm{~min}$, resuspended at a concentration of $1 \times 10^{6} \mathrm{ml}^{-1}$ in RPMI-1640 culture medium containing freshly reconstituted BCG (Danish strain 1331; Statens Serum Institut, Copenhagen, Denmark) vaccine at a concentration of $1 \times 10^{5} \mathrm{CFU} \mathrm{ml}^{-1}\left(10 \mu \mathrm{g} \mathrm{ml}^{-1}\right)$, and incubated for $72 \mathrm{~h}$ in a humidified atmosphere with $5 \% \mathrm{CO}_{2}$ in air at $37^{\circ} \mathrm{C}$. Peripheral-blood mononuclear cells were removed by centrifugation at $200 \mathrm{~g}$ for $10 \mathrm{~min}$ for analysis of immune cell profile. The exhausted medium was then centrifuged at $\sim 16000 \mathrm{~g}$ for $10 \mathrm{~min}$ before storing at $-80^{\circ} \mathrm{C}$, and those from BCG-treated PBMCs were designated as 'BCG supernatants' compared with the 'CONT supernatant', which were from untreated PBMCs.

\section{Defining immune subsets}

Peripheral-blood mononuclear cells were washed twice in wash buffer (PBS containing 1\% (v/v) FBS and 0.09\% (v/v) $\mathrm{NaN}_{3}$ ), and stained for $30 \mathrm{~min}$ at $4{ }^{\circ} \mathrm{C}$ with the following antibodies: peridinin chlorophyll protein anti-CD3, phycoerythrin anti-TCR $\gamma \delta$, fluorescein isothiocyanate (FITC) anti-CD69 and allophycocyaninconjugated anti-CD56 (all from BD Biosciences Ltd, Oxford, UK and used at $1: 1000)$. Matched isotype control antibodies were used to determine background staining. Cells were washed in wash buffer and fixed in $4 \%$ paraformaldehyde (BD Biosciences) for $20 \mathrm{~min}$ at $4{ }^{\circ} \mathrm{C}$. Expressions of the surface markers were analysed using a BD LSR II with dedicated proprietary software (BD Biosciences).

\section{Cytokine analysis of supernatants}

Peripheral-blood mononuclear cells $\left(1 \times 10^{6}\right)$ were plated into 96-well plates, and cultured with BCG $\left(1 \times 10^{5} \mathrm{CFU} \mathrm{m}^{-1}\right)$ for up to $72 \mathrm{~h}$. Plates were centrifuged at $200 \mathrm{~g}$ for $10 \mathrm{~min}$ and supernatants removed. The concentrations of IFN- $\gamma$, TNF- $\alpha$, IL10, IL5, IL4 and IL2 in these PBMC supernatants were determined by using a $\mathrm{Th}_{1} / \mathrm{Th}_{2}$ cytometric bead array (BD Biosciences) according to manufacturer's instructions.

\section{Human leucocyte antigen class I}

Exponentially growing cells were reset in fresh culture medium at $2 \times 10^{5} \mathrm{ml}^{-1}$. Following a settling-in period of $24 \mathrm{~h}$, cells were treated for $48 \mathrm{~h}$ with the PBMC supernatants. To study the impact of inhibiting c-Jun N-terminal kinase (JNK) on HLA1 expression, $10 \mu \mathrm{M}$ of SP600125 (Sigma) was included to the cells with and without the PBMC supernatants. Cells were harvested $\left(1 \times 10^{5}\right)$ and washed twice in wash buffer, and then incubated with anti-HLA1FITC antibody (anti-HLA:ABC 1:1000; BD Biosciences) for $30 \mathrm{~min}$ at $4{ }^{\circ} \mathrm{C}$. Acquisition of data was performed within $1 \mathrm{~h}$ using a FACSCalibur (BD Biosciences). Ten thousand cells were analysed for each sample, and the mean fluorescence intensity (MFI) of HLA1 determined using the program WinMDI v2.9 (http:// facs.scripps.edu/software.html).

To study the role of IFN- $\gamma$ and TNF- $\alpha$ on modulating HLA1 expression on tumours, exponentially growing cells were treated for $24 \mathrm{~h}$ with PBMC supernatants in the presence or absence of anti-IFN- $\gamma$ (AB-285-NA) and anti-TNF- $\alpha$ (AB-210-NA) (both at $10 \mu \mathrm{g} \mathrm{ml}^{-1}$ : R\&D Systems, Oxford, UK). Normal goat IgG was used as the isotype control. Cells were harvested and HLA1 expression determined as described previously.

\section{Cytotoxicity assay}

T-cells were isolated from pathologically healthy donor buffy coats (National Blood Service) using sequential negative cell selection with magnetic beads coated with anti-CD3, followed by positive cell isolation using magnetic bead coated with anti-CD8 (Miltenyi Biotec Ltd, Surrey, UK). CD8 + purities were assessed by flow cytometry, and only those preparations with purities $>90 \%$ were used. Tumour cells pretreated with PBMC supernatants for $48 \mathrm{~h}$ were reset in culture medium at $1 \times 10^{4}$ per well in a 96 -well plate, and allowed to adhere before adding CD8 + T-cells at an effector:target ratio of $20: 1$. Maximum lysis was established by culturing cells with $1 \%$ triton-X. After a 24 -h incubation period, cell-free media were removed for assessment of lactate dehydrogenase (LDH) release using a proprietary assay kit (BioVision Research Products, Mountain View, CA, USA).

Data were presented as \%cytotoxicity, which was calculated from absorbance values at $590 \mathrm{~nm}$ using the following formula:

$$
\% \text { Cytotoxicity }=\frac{A_{\text {target }+ \text { effector }}-\left(A_{\text {target }}+A_{\text {effector }}\right)}{A_{\text {target }+ \text { TritonX }}-A_{\text {target }}}
$$

\section{Immunoblotting analysis}

Cells were harvested; total cellular protein was solubilised in lysis buffer (New England Biolabs, Hitchin, UK) and resolved by Trisglycine electrophoresis using a $4-12 \%$ bis-tris gradient gel. Following transfer of proteins to $0.45 \mu \mathrm{m}$ nitrocellulose membranes, blocking was performed in $5 \%(\mathrm{w} / \mathrm{v})$ non-fat milk in TTBS $(0.5 \%(\mathrm{v} / \mathrm{v})$ Tween-20 in tris buffered saline $(50 \mathrm{mM}$ Tris base with $150 \mathrm{mM} \mathrm{NaCl}$; pH 7.6)). Primary antibody probing was performed with anti-AKT, anti-phospho AKT, anti-ERK, anti-phospho ERK, anti-JNK, anti-phospho JNK, anti-p38 MAPK and anti-phospho p38 MAPK. All primary antibodies were obtained from New England Biolabs and used at a dilution of $1: 1000$, unless stated otherwise. Anti-GAPDH was used as a loading control (1:2000New England Biolabs). Following three washing steps in TTBS, horseradish peroxidase-conjugated antispecies IgG1 was used as the secondary antibody (Amersham Biosciences Ltd, Little 
A

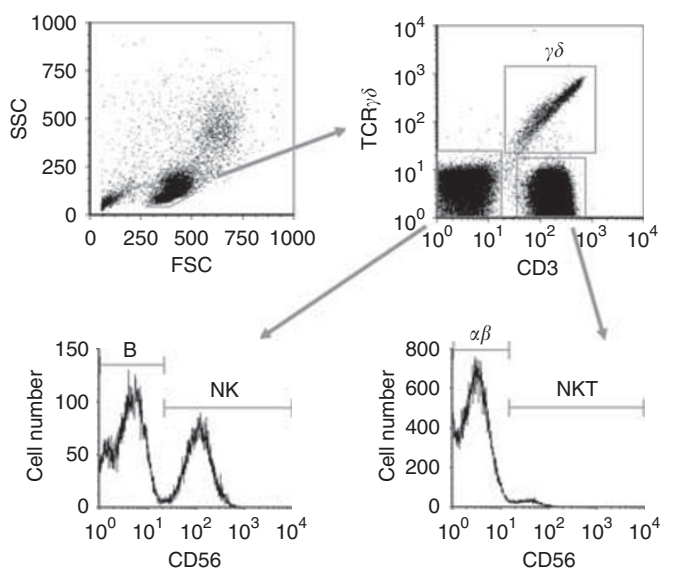

B
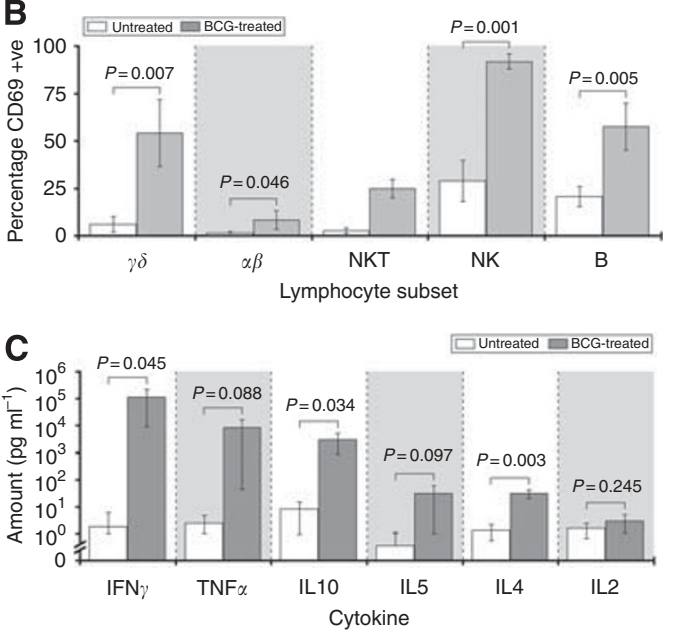

Figure I Effect of BCG on the active status of immune cells and cytokine production. Peripheral-blood mononuclear cells harvested from pathologically normal subjects were exposed to BCG for up to $72 \mathrm{~h}$ before assessing the percentage of CD69-expressing cells by nested flow cytometric analyses. The gating profile $(\mathbf{A})$ allowed for identification of five immune subsets, and the percentage of CD69 positive cells assessed (B). The amounts of six cytokines produced by PBMCs in response to BCG were also assessed $(\mathbf{C})$. Each data column represents the mean and s.d. of five separate individuals at the $72-\mathrm{h}$ time-point, except NKT, where $n=2$ as only $2 / 5$ donors had detectable levels of NKT cells. P-values from paired $t$-tests.

Chalfont, UK). Bands were visualised by the ECL-plus detection system (Amersham).

\section{RESULTS}

\section{BCG alters the activation status of immune cell subsets}

Peripheral-blood mononuclear cells harvested from healthy subjects were exposed to BCG for up to $72 \mathrm{~h}$ before assessing the activation status of each of the key immune effector subsets by flow cytometry. Activation was indicated by CD69 expression, and our gating strategy is defined in Figure 1A. Results showed that BCG was capable of increasing the percentage of CD69 expression in all the cell types studied, and that the increase was apparent as early as $6 \mathrm{~h}$. Data for $72 \mathrm{~h}$ are shown in Figure 1B. Furthermore, the effect of BCG on CD69 expression on each of the cell types was robust and consistent in all five replicates.

\section{BCG stimulates cytokine production}

The effect of culturing PBMCs with BCG on a number of $\mathrm{Th}_{1} / \mathrm{Th}_{2}$ cytokines was assessed by cytometric bead analysis, and results showed that BCG generally increased the amount of cytokines detected in the supernatants. This effect was apparent as early as $6 \mathrm{~h}$, and data for the $72 \mathrm{~h}$ time-point is shown in Figure 1C. Results suggested a $\mathrm{Th}_{1}$-biased effect of BCG; specifically, although there were significant increases in the majority of the cytokines assessed, the extents to which IFN- $\gamma$, TNF- $\alpha$ and IL10 were elevated were greater than for IL5, IL4 and IL2. For example, at $72 \mathrm{~h}$, although BCG resulted in significant increases in both IFN- $\gamma$ and IL4 compared with untreated controls, the former was increased to a concentration of $\sim 115000 \mathrm{pg} \mathrm{ml}^{-1}$, while the latter was increased to $\sim 32 \mathrm{pg} \mathrm{ml}^{-1}$. Furthermore, the concentrations of these two cytokines were similar in unstimulated control PBMCs (1.8 vs $1.4 \mathrm{pg} \mathrm{ml}^{-1}$, respectively).

\section{BCG supernatant increases HLA1 expression on some tumour cells}

The expression of HLA1 was assessed by flow cytometry using a proprietary antibody directed against HLA-ABC and presented as
MFIs. Our results confirmed that basal HLA1 expression varied in the cell lines (Liu et al, 2010). The order of expressions were KM $<$ A549 < MIAPaCa2 < MCF7 < HCT116 (MFI normalised to isotype control: $0.91 \pm 0.0076 ; \quad 7.5 \pm 0.61 ; 9.1 \pm 0.93 ; \quad 34 \pm 2.7$; $41 \pm 3.9$, respectively). There was little effect on HLA1 expression of culturing KM and MIAPaCa2 cells with BCG supernatant. However, in A549, HCT116 and MCF7 cells culturing with BCG supernatant caused significant increases in HLA1 expressions (Figure 2A); for example, mean MFI in MCF7 cells treated with BCG supernatant was 760 vs 290 in CONT-supernatant-treated cells $(P<0.02)$. For clarity, data were also presented relative to the CONT supernatants (Figure $2 \mathrm{~B}$ ).

\section{HLA1 expression is associated with cytolytic T-cell function}

Allogeneic CD8 + T-cells were harvested from pathologically normal subjects and admixed with tumour cells pre-exposed to PBMC supernatants. The extent of cell death was established by assessing LDH activity in the medium by using a proprietary kit. Results indicated significant increases in the percentage of cytotoxicity following treatment with BCG supernatant only in those tumour cells with increased HLA1 expression (Figure 2C). For example, cytotoxicity in MCF7 cells was increased from a basal level of just $1.0 \pm 0.42 \%$ to $19 \pm 3.0$ when BCG supernatant was used $(P=0.008)$.

\section{BCG supernatants alter the expressions of important signalling molecules}

To investigate whether the changes in HLA1 expression and/or cytotoxic responses could be associated with changes in intracellular signalling pathways, whole cell lysates obtained from the five cells cultured with supernatants were analysed by immunoblotting. A broad panel of proteins representing key protagonists in a number of signalling cascades downstream of receptor tyrosine kinases was surveyed. The intention was to employ this approach to identify a signalling protein most likely to trigger the phenotypic changes. Results highlighted differences in the cellular responses between the cells exposed to supernatants. In those cell lines where HLA1 was shown to be unaffected by BCG supernatant, there were no clear changes to the expressions of the signalling molecules studied (Figure 3A). However, in those cells where HLA1 was 

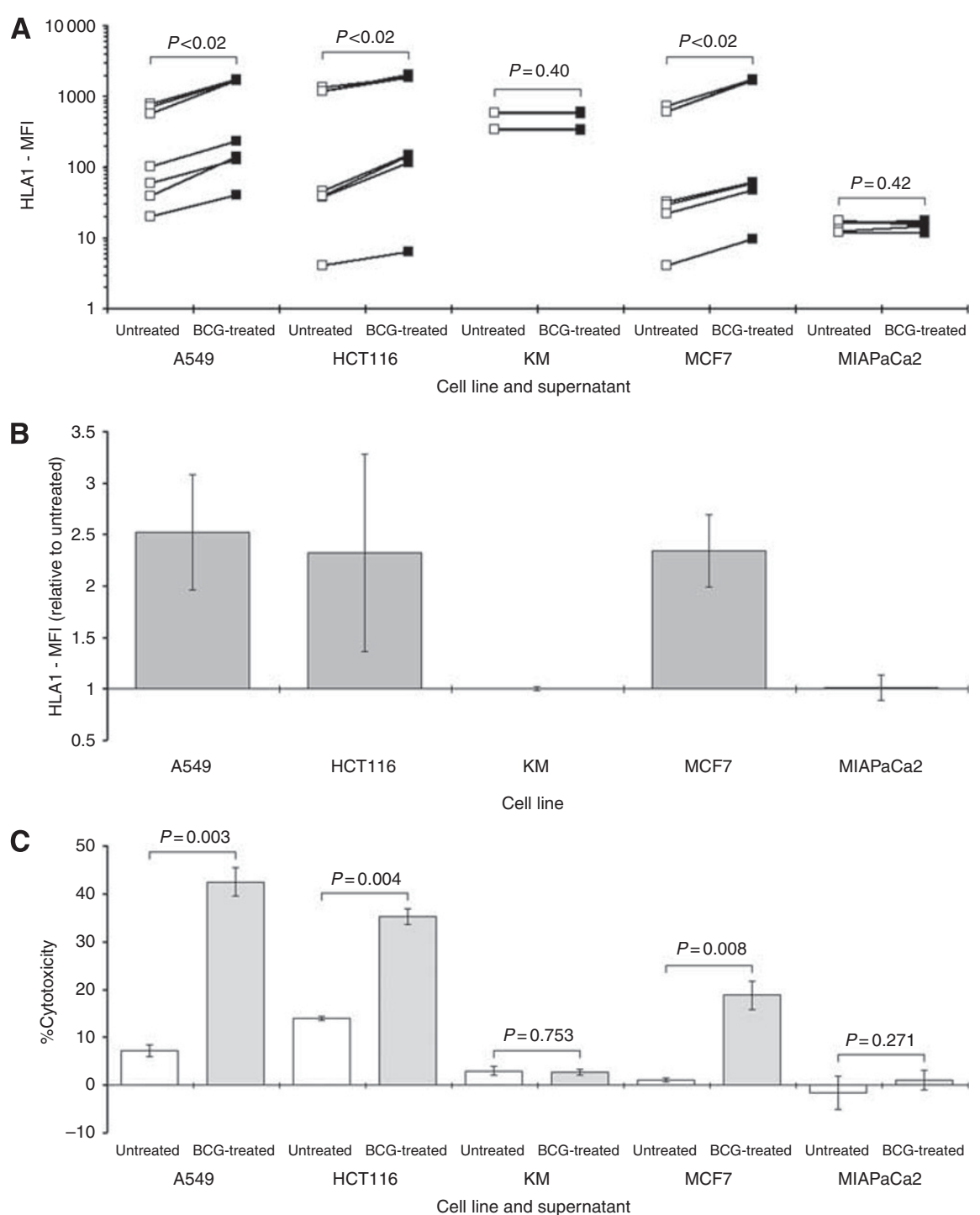

Figure 2 Effect of supernatants on HLAI expression in tumour cells. Five tumour cell lines were cultured for $24 \mathrm{~h}$ with the supernatants derived from PBMCs treated with BCG (BCG-supernatant), before assessing HLAI expression on the tumour cells. The basal levels of HLAI expression expressed as MFI relative to the isotype control are presented in the text. The magnitudes of these expressions were compared with those on tumours after stimulation with supernatants from untreated PBMCs (CONT-supernatant). Data were presented as raw MFI (A) or relative to the respective controls (B). The relationship between HLAI expression and cytotoxic effect of allogeneic CD8 + T-cells were assessed by measuring LDH activity $(\mathbf{C})$. Effector: target ratios were at 20: I and each data column represented the mean and s.d. of at least three separate experiments. P-values were from paired tests.

affected by BCG supernatants, there were changes seen (Figure 3B). Generally, there were small changes to protein expressions; however, the clearest increases were observed in JNK signalling, which happened to be common to two of the three cell lines studied (Figure 3B). Significant increases were also seen to the phospho expressions of AKT in MCF7 and p38 MAPK in A549.

\section{Disrupting JNK signalling affects HLA1 upregulation by BCG supernatant}

To assess the impact of JNK signalling on HLA1 expression, we cultured HCT116 and MCF7 cells with SP600125, an anthrapyrazolone inhibitor of JNK (Bennett et al, 2001), and assessed the extent to which HLA1 expression was altered by BCG supernatant. Early results showed that treating cells with SP600125 did not completely reverse the HLA1-enhancing effect of the BCG supernatant. However, results showed that culturing cells in the presence of SP600125 caused a significant reduction in the ability of BCG supernatant to enhance HLA1 expression in the cells (Figure 4A-C). For example, an active concentration of SP600125 reduced the increase in HLA1 expression in MCF7 cells caused by BCG supernatant from 7.4-fold to 5.4-fold (Figure 4B).

\section{Neutralising IFN- $\gamma$ and TNF- $\alpha$ affects HLA1 upregulation by BCG supernatant}

To explore the effects of IFN- $\gamma$ and TNF- $\alpha$ in BCG supernatants on modulating HLA1 expression on A549, HCT116 and MCF7 cells, their activities were disrupted by using antibodies against them. Results showed that these neutralising antibodies significantly reversed the HLA1-enhancing effects of the BCG supernatant in the three cell lines tested (Figure 5A). Furthermore, the enhanced cytotoxicity afforded by $\mathrm{T}$-cells that was associated with increased HLA1 expression was negated by this co-culture with the neutralising antibodies (Figure 5B). 
A KM AKT $\quad$ ERK $\quad$ JNK $\quad$ P38

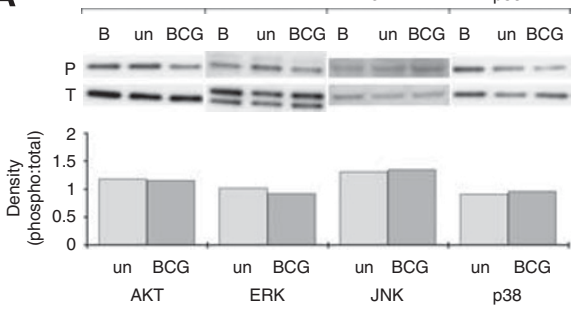

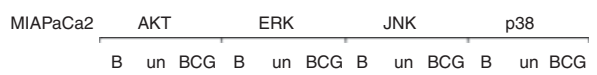

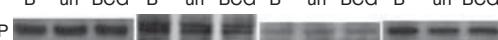

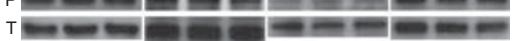

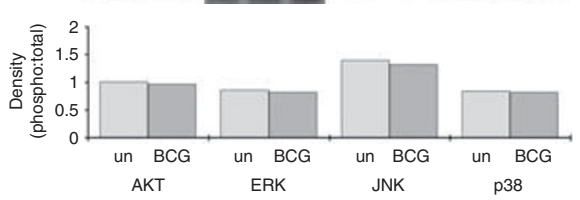

$B^{\text {AKT }}$

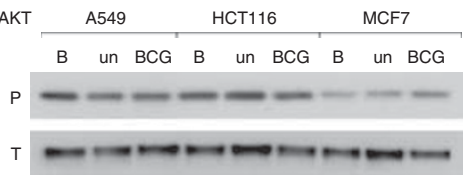

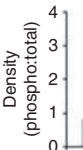

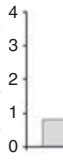

JNK

NK $\quad$ A549

$B$ un $B C G$ un $B C G$ un $B C G$
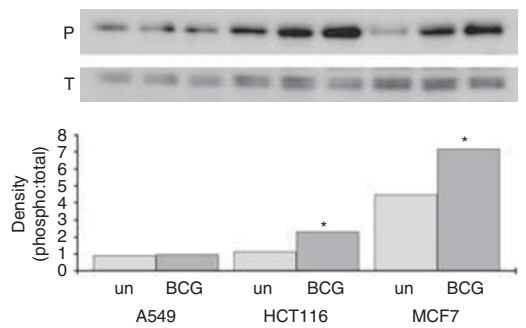

Figure 3 Effect of supernatants on intracellular signalling proteins. Cells were cultured with BCG supernatants for $24 \mathrm{~h}$ before western blotting for the proteins indicated. AKT, ERK, JNK and p38 MAPK were the proteins assessed as they represented a broad range of signalling elements indicating receptor activation and intracellular functioning. Samples were designated 'BCG' if treated with BCG supernatants, and expressions were compared with those from tumours cultured with CONT supernatants ('un') and basal expressions ('B': where tumours were cultured in standard medium). Both the phosphorylated $(P)$ and total $(T)$ levels were assessed, and densitometry were performed showing the effect of supernatants on the phospho: total ratio. Cell lines that were unaffected by BCG supernatants with regard to HLAI expression are shown in $(\mathbf{A})$, while those exhibiting increases in HLAI expression following treatment in $B C G$ supernatant are shown in $(\mathbf{B}) .{ }^{*} P<0.05$ when compared with the untreated control as determined by paired $t$-tests.
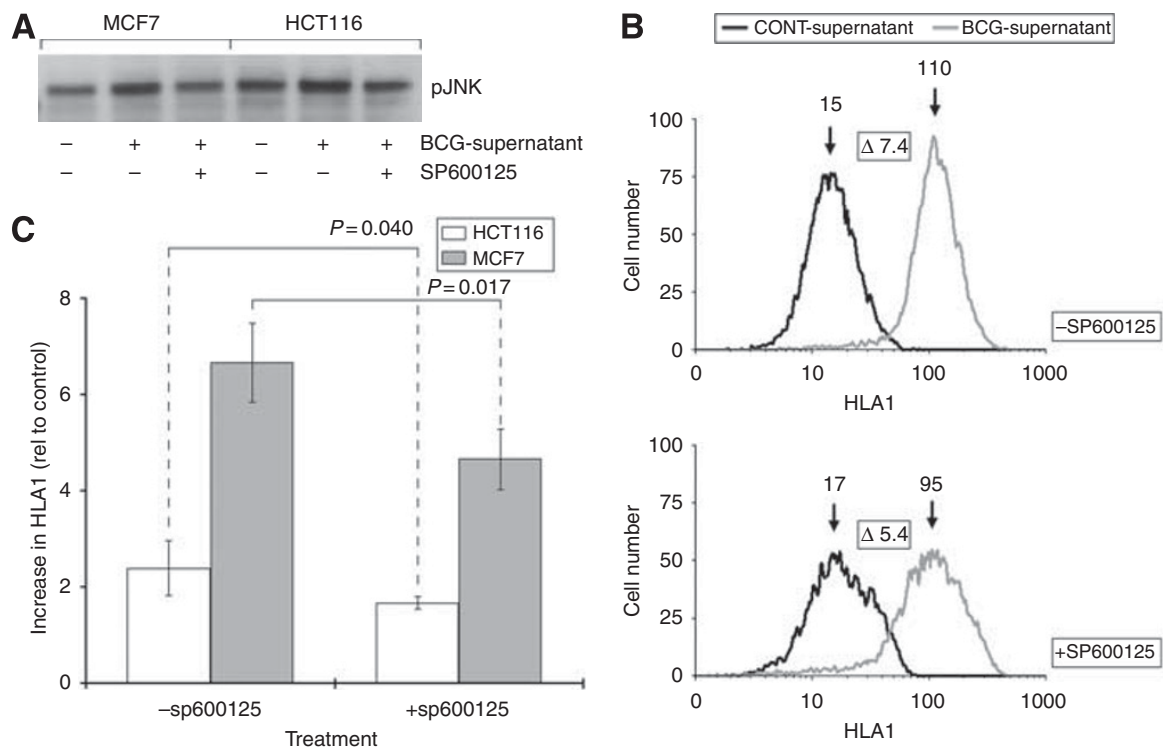

Figure 4 Effect of JNK inhibition on HLAI expression. HCTII6 and MCF7 cells were treated with BCG supernatant $+/-$ the JNK inhibitor SP600 I25, and the effect of reduced JNK action on HLAI expression assessed. The increase in phosphorylated JNK induced by BCG supernatant was negated by SP600I 25 (A), and the ability of BCG supernatant to enhance HLAI expression compromised by JNK inhibition (B and $\mathbf{C})$. Representative histograms from MCF7 experiments are shown (B), and each data column shows means and s.d.'s of a minimum of four separate experiments.

\section{DISCUSSION}

This study was undertaken as part of our larger remit to investigate the role of immunotherapies in an oncological setting, with particular interest in understanding the value of combining them with other modalities. These combinations could include agents that both directly and indirectly contributed to an improved action. In the current study, we specifically investigated the immunopotentiating effects of BCG, and explored their direct effects on PBMCs, as well as the effects that supernatants derived from these preparations could have on tumour cells directly. In summary, our results showed that BCG potently increased the activity of a number of immune subsets, which lead to significant potentiation of the cytokine output of these cells. Additionally, the supernatants in which they were found significantly increased HLA1 expression on a number of tumour cell lines. Increased immunovisibility was dependent upon these cytokines and associated with enhanced cytolytic T-cell activity. Moreover, the 

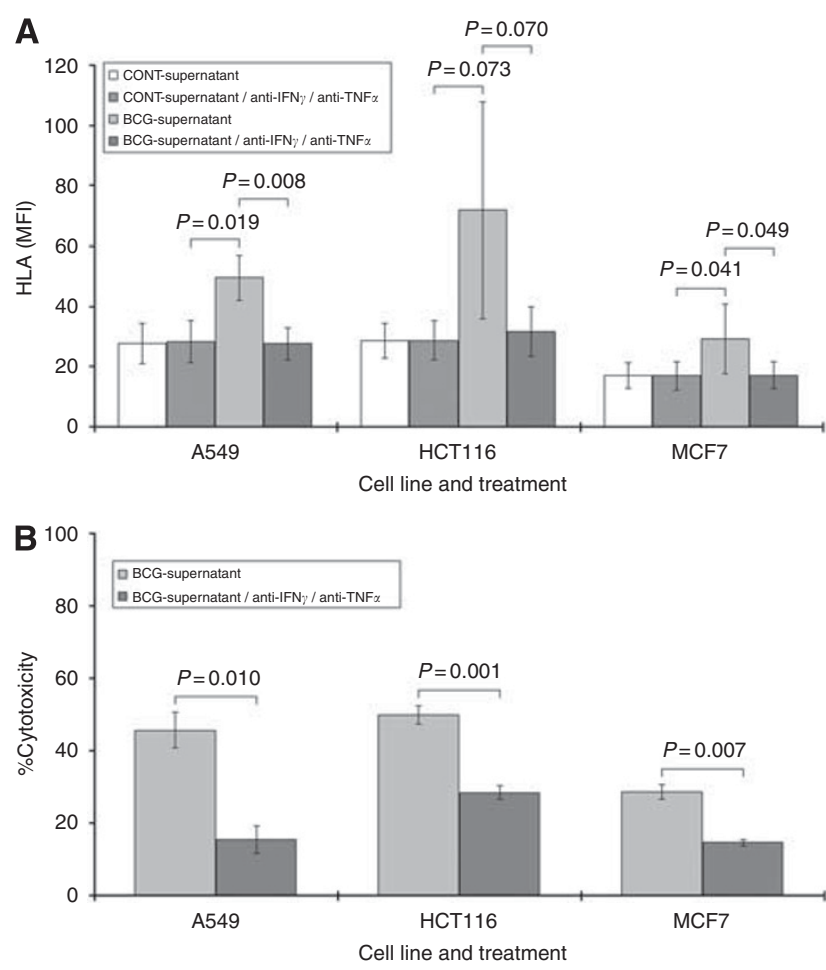

Figure 5 Effect of anti-IFN- $\gamma$ and anti-TNF- $\alpha$ on HLAI expression. A549, HCTI I 6 and MCF7 cells were cultured with BCG supernatant in the presence or absence of neutralising antibodies against IFN- $\gamma$ and TNF- $\alpha$. Antagonising these cytokines caused reversal of the increases in HLAI seen as a consequence of BCG-supernatant culture (A). Furthermore, culturing with the neutralising antibodies disrupted the extent of cytotoxicity seen with admixing with CD8 + T-cells $(\mathbf{B})$. Each data column represents the mean and s.d. of at least three separate experiments.

ability of tumour cells to upregulate HLA1 was partially mediated by JNK signalling.

A large number of malignant diseases are associated with suppression of cell-mediated immune responses, but humoral immunity remains unchanged or is increased (Dalgleish and O'Byrne, 2002). This presents the interesting notion that oncogenesis can sabotage cell-mediated immunity by biasing the $T h_{1}-/ \mathrm{Th}_{2^{-}}$ response towards a $\mathrm{Th}_{2}$-dominant immune profile that supports cancer growth (De Monte et al, 2011). We and others have suggested that adjusting this 'immune rheostat' to a $\mathrm{Th}_{1}$-dominant setting can restore potent cell-mediated immunity, and result in objective tumour responses (Heriot et al, 2000; Dalgleish and O’Byrne, 2002). These approaches could include BCG, which is known to alter the profile of cytokine output of PBMCs (Alexandroff et al, 1999; Luo et al, 2003). For that reason, in the first part of our study, we cultured freshly harvested PBMCs from pathologically normal individuals, and assayed their responses to BCG vaccine used at a clinically achievable concentration $\left(1 \times 10^{5} \mathrm{CFU} \mathrm{m} \mathrm{m}^{-1}\right)$. Our results showed that BCG significantly increased the percentage of cells expressing the early activation marker CD69, and that these increases were seen in all the immune subsets surveyed. Subsequent analysis of the cytokines released into the supernatants from PBMCs following BCG treatment revealed higher levels of IFN- $\gamma$ in comparison with IL4, suggestive of a $\mathrm{Th}_{1}$-predominant response supportive of antitumour action. Although the specific source of these cytokines was not identified in the current study, we have previously seen significant increases in intracellular levels of IFN- $\gamma$, TNF- $\alpha$ and IL2 in CD3 + CD4 + cells following stimulation with BCG and other mycobacteria preparations (data not shown). Nevertheless, what was clear was a clear and significant increase in the $\mathrm{Th}_{1}$-cytokines IFN- $\gamma$ and
TNF- $\alpha$ that are profoundly associated with the regulation of HLA1 (Seliger et al, 2008), which highlights another facet of BCG that is exploitable therapeutically.

Manipulating tumour HLA1 expression as a means of potentiating the efforts of cytotoxic effectors is complex. There appears to be a fine balance in which levels are kept low to ensure evasion of CD8 + T-cells surveillance. However, loss of HLA1 opens the door to innate immune responses involving NK cells, which serve to clear cells that are devoid of or low in HLA1 (Du and Wang, 2011). Thus, restoring HLA1 expression in this way could inadvertently have the opposite effect of protecting cells from therapy by rendering tumour cells refractory to $\mathrm{NK}$ cell-mediated lysis (Storkus et al, 1991). These considerations need to be made when manipulating HLA1 expression, but it is worth noting that selective pressures on tumours results in reductions rather than increases in HLA1, which suggests that evasion of the adaptive response may be the more favourable outcome (Cabrera et al, 2003; Aptsiauri et al, 2007). These reductions can be caused by the loss of transcription and translation elements (Garrido et al, 1993) or epigenetic modifications that silence regulatory genes (Tomasi et al, 2006). Losses can also result from dysregulations of cellular signalling pathways, which are commonly altered in cancer cells, as well as from reduced responsiveness to cytokines (Rodríguez et al, 2007). Therefore, therapeutic interventions that can counter these oncogenic transformations and/or restore immunovisibility would be of value.

We have previously reported similar HLA1-modulating actions of some chemotherapies (Liu et al, 2010), and so adopted similar models to investigate the effect that the exhausted media from PBMCs cultured with BCG could have on HLA1 expression in a panel of tumour cell lines. Results showed HLA1 to be significantly increased in a number of cell lines, some of which had intrinsically low starting levels, and that these enhancements were associated with clear improvements in the cytotoxic actions of allogeneic CD8 + T-cells. Our results thus support the notion that BCG supernatants can increase HLA1 expression on tumour cells, and therefore, indirectly enhance tumour clearance. While it is already known that BCG can stimulate PBMCs to produce IFN- $\gamma$ (Luo et al, 2003), and that recombinant IFN- $\gamma$ can enhance HLA1 expression (Seliger et al, 2008), our data unite these two concepts by highlighting the ability of supernatants from BCG-treated PBMCs to boost HLA1 expression directly. Furthermore, the basal levels of HLA1 expressions on tumours do not directly influence their ability of being upregulated by these BCG supernatants. The tantalising possibility that other mycobacteria possess similar HLA1-boosting effects is currently being explored.

We next assessed the impact of BCG supernatants on four molecules downstream of cytokine receptors, which represented key components of intracellular signalling involved in the growth and development in cancer cells (Sebolt-Leopold and English, 2006). Our intention was to identify components that were common to the cell lines tested and not to fully deconvolute complex interplay between the cascades. Of particular interest was JNK, which was altered in two of the three cell lines studied. Antagonising JNK activity in tumour cells exposed to BCG supernatant significantly interfered with its ability to increase HLA1, which supported the important role of JNK signalling and HLA1 expression in tumour cells. Moreover, the JNK pathway has a prominent role in regulating HLA1 expression in tumour cells, whose activity is determined by IFN- $\gamma$ signalling (Bach et al, 1997). In support of the observations on JNK signalling, we also explored further the roles of IFN- $\gamma$ and TNF- $\alpha$ in increasing HLA1 expression on tumours. Our results showed that antagonising the actions of these cytokines by using neutralising antibodies against them negated the increase in HLA1 expression caused by BCG supernatant. Moreover, the enhanced cell death associated with increased HLA1 was also lost (Figure 5). This has been seen previously in the context of melanoma, where disrupting IFN- $\gamma$ signalling can adversely affect HLA1 functionality in tumours 
(Rodríguez et al, 2007; Respa et al, 2011). Parenthetically, the melanoma cell line KM that was used in our study was refractory to BCG supernatant, and moreover, culturing them directly with active doses of recombinant IFN- $\gamma$ had no effect on HLA1 expression (data not shown). This suggests differences in the mechanisms of these cytokines in modifying HLA1 that are cell line dependent. Further work to deconvolute these signalling pathways is ongoing, and will go someway in helping to identify drug partners for combination therapies, which may vary and be patient/tumour specific.

In summary, studies have suggested that the induction of an effective immune response could assist in the elimination of tumour. Approaches have included using DC vaccines to stimulate $\mathrm{T}$-cell responses, and potentiating the cytolytic actions of T-cells through the use of cytokines (Copier et al, 2011). However, to date, successes have been limited. In spite of this, one area that has gained momentum recently is the use of conventional chemotherapy as immune stimulators. This exploits the multiple mechanisms of their action and utilises the immune-related effects of some chemotherapies that support a cell-mediated effect. For this reason, competent T-cell and NK cell functions are understandable pre-requisites to maximising the benefits of this approach. Therefore, individuals with a compromised immune system, or those possessive of one that is biased towards a $\mathrm{Th}_{2}$-response, may not benefit as much. For this reason, employing BCG as an adjuvant to these chemotherapies could be clinically useful for two reasons; first, by restoring the immune rheostat to an antitumour setting, and second, by restoring immune visibility of tumours via the HLA1-enhancing properties. Taken together, these data support a combination strategy, in which chemotherapy is supplemented with systemic immune stimulation, and that this approach would be a powerful tool in cancer therapy.

\section{ACKNOWLEDGEMENTS}

This work was only possible through the continued support of Celgene Corp., USA and the Cancer Vaccine Institute, UK.

\section{REFERENCES}

Abbas AK, Murphy KM, Sher A (1996) Functional diversity of helper T lymphocytes. Nature 383: 787-793

Alexandroff AB, Jackson AM, O'Donnell MA, James K (1999) BCG immunotherapy of bladder cancer: 20 years on. Lancet 353: $1689-1694$ Aptsiauri N, Cabrera T, Mendez R, Garcia-Lora A, Ruiz-Cabello F, Garrido F (2007) Role of altered expression of HLA class I molecules in cancer progression. Adv Exp Med Biol 601: 123-131

Bach EA, Aguet M, Schreiber RD (1997) The IFN gamma receptor: a paradigm for cytokine receptor signaling. Annu Rev Immunol 15: 563-591

Bennett BL, Sasaki DT, Murray BW, O'Leary EC, Sakata ST, Xu W, Leisten JC, Motiwala A, Pierce S, Satoh Y, Bhagwat SS, Manning AM, Anderson DW (2001) SP600125, an anthrapyrazolone inhibitor of Jun N-terminal kinase. Proc Natl Acad Sci 98: 13681-13686

Cabrera T, López-Nevot MA, Gaforio JJ, Ruiz-Cabello F, Garrido F (2003) Analysis of HLA expression in human tumor tissues. Cancer Immunol Immunother 52: 1 -9

Copier J, Bodman-Smith M, Dalgleish A (2011) Current status and future applications of cellular therapies for cancer. Immunotherapy 3: 507-516

Dalgleish AG, O'Byrne KJ (2002) Chronic immune activation and inflammation in the pathogenesis of AIDS and cancer. Adv Cancer Res 84: 231-276

De Monte L, Reni M, Tassi E, Clavenna D, Papa I, Recalde H, Braga M, Di Carlo V, Doglioni C, Protti MP (2011) Intratumor T helper type 2 cell infiltrate correlates with cancer-associated fibroblast thymic stromal lymphopoietin production and reduced survival in pancreatic cancer. J Exp Med 208: $469-478$

Du C, Wang Y (2011) The immunoregulatory mechanisms of carcinoma for its survival and development. J Exp Clin Cancer Res 30: 12

Dunn GP, Bruce AT, Ikeda H, Old LJ, Schreiber RD (2002) Cancer immunoediting: from immunosurveillance to tumor escape. Nat Immunol 3: $991-998$

Ellyard JI, Simson L, Parish CR (2007) Th2-mediated anti-tumour immunity: friend or foe? Tissue Antigens 70: 1-11

Garrido F, Cabrera T, Concha A, Glew S, Ruiz-Cabello F, Stern PL (1993) Natural history of HLA expression during tumour development. Immunol Today 14: 491-499

Heriot AG, Marriott JB, Cookson S, Kumar D, Dalgleish AG (2000) Reduction in cytokine production in colorectal cancer patients: association with stage and reversal by resection. $\mathrm{Br} J$ Cancer 82: $1009-1112$

Hrouda D, Baban B, Dunsmuir WD, Kirby RS, Dalgleish AG (1998) Immunotherapy of advanced prostate cancer: a phase I/II trial using Mycobacterium vaccae (SRL172). Br J Urol 82: 568-573
Liu WM, Dennis JL, Fowler DW, Dalgleish AG (2011) The gene expression profile of un-stimulated dendritic cells can be used as a predictor of function. Int J Cancer, e-pub ahead of print 31 March 2011, doi:10.1002/ ijc. 26101

Liu WM, Fowler DW, Smith P, Dalgleish AG (2010) Pre-treatment with chemotherapy can enhance the antigenicity and immunogenicity of tumours by promoting adaptive immune responses. Br J Cancer 102: 115-123

Luo Y, Chen X, O'Donnell MA (2003) Role of Th1 and Th2 cytokines in BCG-induced IFN-gamma production: cytokine promotion and simulation of BCG effect. Cytokine 21: 17-26

Maraveyas A, Baban B, Kennard D, Rook GA, Westby M, Grange JM, Lydyard P, Stanford JL, Jones M, Selby P, Dalgleish AG (1999) Possible improved survival of patients with stage IV AJCC melanoma receiving SRL 172 immunotherapy: correlation with induction of increased levels of intracellular interleukin-2 in peripheral blood lymphocytes. Ann Oncol 10: $817-824$

Nicholson S, Guile K, John J, Clarke IA, Diffley J, Donnellan P, Michael A, Szlosarek P, Dalgleish AG (2003) A randomized phase II trial of SRL172 (Mycobacterium vaccae) $+/-$ low-dose interleukin-2 in the treatment of metastatic malignant melanoma. Melanoma Res 13: 389-393

Orme IM, Andersen P, Boom WH (1993) T cell response to Mycobacterium tuberculosis. J Infect Dis 167: $1481-1497$

Respa A, Bukur J, Ferrone S, Pawelec G, Zhao Y, Wang E, Marincola FM, Seliger B (2011) Association of IFN-\{gamma\} signal transducti. Clin Cancer Res 17: 2668-2678

Rodríguez T, Méndez R, Del Campo A, Jiménez P, Aptsiauri N, Garrido F, Ruiz-Cabello F (2007) Distinct mechanisms of loss of IFN-gamma mediated HLA class I inducibility in two melanoma cell lines. BMC Cancer 7: 34

Sebolt-Leopold JS, English JM (2006) Mechanisms of drug inhibition of signalling molecules. Nature 441: 457-462

Seliger B, Ruiz-Cabello F, Garrido F (2008) IFN inducibility of major histocompatibility antigens in tumors. Adv Cancer Res 101: 249-276

Storkus WJ, Salter RD, Alexander J, Ward FE, Ruiz RE, Cresswell P, Dawson JR (1991) Class I-induced resistance to natural killing: identification of nonpermissive residues in HLA-A2. Proc Natl Acad Sci 88: $5989-5992$

Tomasi TB, Magner WJ, Khan AN (2006) Epigenetic regulation of immune escape genes in cancer. Cancer Immunol Immunother 55: 1159-1184

Yang J, Yi Q (2010) Killing tumor cells through their surface beta(2)microglobulin or major histocompatibility complex class I molecules. Cancer 116: $1638-1645$

This work is published under the standard license to publish agreement. After 12 months the work will become freely available and the license terms will switch to a Creative Commons Attribution-NonCommercial-Share Alike 3.0 Unported License. 